\section{Potential for oral oligofructose- enriched inulin in the treatment of ulcerative colitis}

Casellas et al. have conducted a prospective double-blind, placebo-controlled trial to investigate the anti-inflammatory potential of the prebiotic Synergy 1 (an oligofructose-enriched inulin) in patients with ulcerative colitis. Nineteen patients who presented with a relapse of mildly to moderately active ulcerative colitis were randomly allocated to receive oral Synergy 1 or placebo (both at $4 \mathrm{~g}$ three times daily) in addition to standard mesalazine therapy ( $3 \mathrm{~g} /$ day) and a low-fiber diet for 14 days.

One participant withdrew from the study before starting treatment, and three (one from the placebo group and two from the prebiotic group) withdrew by the end of the first week because their disease activity increased. The prebiotic was well tolerated and no notable side effects were reported. On study completion, all patients in the prebiotic group had a significant reduction in the levels of fecal calprotectin, a noninvasive marker of intestinal inflammation, and were in clinical remission; in the placebo group, the reduction was not significant and two patients still had clinically active disease. Neither group had a change in the levels of fecal human DNA, another noninvasive marker of intestinal inflammation.

The authors conclude that Synergy 1 seems to safely and effectively mitigate intestinal inflammation in patients with ulcerative colitis and thereby improves the response to mesalazine. They recommend that larger studies be performed to evaluate the ability of Synergy 1 to induce or maintain remission of active ulcerative colitis.

Original article Casellas F et al. (2007) Oral oligofructoseenriched inulin supplementation in acute ulcerative colitis is well tolerated and associated with lowered faecal calprotectin. Aliment Pharmacol Ther 25: 1061-1067

\section{Perturbations in infant gut microbiota composition precede development of childhood atopy}

Emerging evidence now suggests that the dramatic increases in the prevalence of childhood atopy in Western countries in recent decades result from Western diet and antibiotic use, rather than from reduced exposure to infections.
Diet and antibiotics could perturb infant gut microbiotic composition, resulting in disruption of normal development of immune tolerance.

In this prospective, national study of 957 eligible infants (whose mothers were participants in the KOALA study), Penders et al. aimed to determine whether the presence or load of colonizing bacteria in 1-month-old infants (as determined by quantitative real-time polymerase chain reaction analysis of fecal samples) was associated with atopic symptoms in children aged up to 2 years. Parents provided questionnaire information on atopic symptoms and potential confounders at study enrollment (34 weeks' gestation) and months 3,7 , 12 and 24 postpartum. Trained nurses took blood samples from, and performed a physical examination for atopic dermatitis in, the 607 24-month-old-infants available for home visit.

After adjustment for confounders (subcohort, parental and sibling atopic history, age at fecal sample collection, infant's sex), the presence of Clostridium difficile was associated with a higher risk of all atopic outcomes: eczema (odds ratio $[O R]$ 1.4), recurrent wheeze (OR 1.75), allergic sensitization (OR 1.54) and atopic dermatitis (OR 1.73). The presence of Escherichia coli was associated with a higher risk of eczema (OR 1.87); this risk increased with increasing count.

The findings provide support for a causal relationship between disturbances in infant gut microbiota composition and subsequent development of atopy.

Original article Penders J et al. (2007) Gut microbiota composition and development of atopic manifestations in infancy: the KOALA Birth Cohort Study. Gut 56: 661-667

\section{Confocal endomicroscopy provides a virtual biopsy of the colon}

A new study has demonstrated that confocal endomicroscopy (CEM), which combines confocal microscopy with traditional endoscopy, provides information on intestinal histopathology comparable to that generated by histopathological images of normal biopsies. In addition, CEM can also provide information on the physiological functions of the mucosa.

Odagi et al. performed CEM in 45 patients and compared the images generated with the histopathological images of 24 normal biopsies: 46 